\title{
Assessment of avian occurrence in the Brazilian chaco
}

\begin{abstract}
This study shows the magnitude of the avifauna occurring in the Brazilian Chaco biome, located in the central region of South America. The Chaco formation is present in Brazil, Paraguay, Bolivia and Argentina, comprising a large phyto physiognomy of nearly $800,000 \mathrm{~km}^{2}$, of which $9,000 \mathrm{~km}^{2}$ is located in Brazil, near the town of Porto Murtinho, a transition zone of the Pantanal wetland. It is formed by spiny trees which lose their small leaves during the dry season. During our field work we registered 326 species of wild birds, and, when added to other published surveys, this number totalizes 355 species living within the Brazilian Chaco. The most common species were Tyrannidae (13.2\%), followed by Thraupidae $(9.2 \%)$ and Icteridae $(4.9 \%)$. Fourteen officially listed threatened bird species were detected during our field work. Our results contribute to the knowledge on bird diversity living in the Brazilian Chaco, and to the potential attraction for sustainable tourism, as an incentive to protect the avifauna and their habitats, achieving conservation for the biodiversity of the region.
\end{abstract}

Volume 2 Issue 4 - 2017

\author{
Maristela Benites, Simone Mamede, Guto \\ Carvalho, Cleber JR Alho \\ Analysis and Conservation of Biodiversity Wildlife, Brazil
}

Correspondence: Cleber JR Alho, Análise e Conservação da Biodiversidade Manejo da Fauna Silvestre Diagnóstico e Avaliação de Impactos Ambientais Lattes, Brazil, Email alhocleber@gmail.com

Received: April 30, 2017| Published: October 18, 2017

Keywords: avifauna, birdwatching, chaco, conservation, threatened bird species

\section{Introduction}

The Chaco is one of the largest biomes in South America, rich in biodiversity, but facing increasing environmental disruptions due to demographic expansion and unsustainable use of natural resources. Wild species of birds can attract tourism as one element to conserve nature, providing economic incentives since biodiversity can represent important socioeconomic and environmental activities. ${ }^{1,2}$ The Chaco is an arid subtropical biome in the center of South America, dominated by savanna vegetation and rare low forest. It is the second largest biome in South America. ${ }^{3}$ This steppe Savanna vegetation formation, as it is known, comprises $520,000 \mathrm{~km}^{2}(46 \%)$ in Argentina, $230,000 \mathrm{~km}^{2}(32 \%)$ in Paraguay, $90,000 \mathrm{~km}^{2}(15 \%)$ in Bolivia and $9,000 \mathrm{~km}^{2}(7 \%)$ in Brazil. There is a predominance of xenomorphic vegetation, with deciduous trees, cacti and bromeliads. ${ }^{3-6}$ The vast Quaternary plain between the Paraguay Parana rivers and the foothills of the western ranges, known as the 'Gran Chaco', is a natural region, $700 \mathrm{~km}$ wide in the east west direction and roughly $1,500 \mathrm{~km}$ from north to south, comprising an area estimated to range from 800,000 to $1,000,000 \mathrm{~km}^{2} .3,4,7$

In its extreme eastern portion is the Paraguay River in Brazilian territory, ${ }^{3}$ south of the Pantanal wetland, in the sub regions Porto Murtinho and Nabileque, and this comprises the wet Chaco. ${ }^{5,8}$ It is a transition area among the surrounding biomes of the Pantanal wetland, namely the Cerrado (savanna), and the Dry Forests there is an interception among the vegetation formations, forming ecotones that cannot easily be identified by sight. There is high density of the palm tree "caranda" (Copernicia alba Morong), and other trees such as "paratudo" (Handroanthus aureus Mattos) and "piuva" (H. heptaphylla (Vell.) Mattos). ${ }^{3,6}$ The Chaco forest in north eastern Argentina and Paraguay, entering Brazilian territory, near the city of Porto Murtinho, is dominated by a hardwood tree red quebracho (Schinopsis balansae Engl) which is typical of this region. ${ }^{3,8}$ The Brazilian Chaco has lately received increasing research attention, especially in plant sciences, but there have been few studies on wild birds. Some studies have even stressed the lack of avian surveys for that region. ${ }^{9,10}$ The last survey carried out in the Brazilian Chaco region reached 282 bird species. ${ }^{11}$ This list of species includes previously collected secondary data. ${ }^{9,12}$ The aim of the present study is to contribute to the knowledge of the rich and diverse avifauna of the Brazilian Chaco region, to pursue conservation and to attract tourists to the region, and thus to implement sustainable use through tourism and birdwatching activities.

\section{Materials and methods}

\section{Study area}

This study was conducted throughout the Chaco region of Porto Murtinho, in the southwestern part of the state of Mato Grosso do Sul, in Brazil. ${ }^{3}$ The regional climate is hot and dry, with annual average rainfall of $970.3 \mathrm{~mm}$ and temperature of $25^{\circ} \mathrm{C}$, with a rainy season from November to February. ${ }^{13}$ The environmental seasonality within this region is more marked by rainfall than by change in temperature. Different kinds of habitats were surveyed, including riparian forest, open dry and flooded fields, savanna (Cerrado) and steppe savanna (Chaco), forest and areas under human use, such as pastures (Figure 1).

\section{Field surveys}

Short term and intensive bird surveys were carried out in the field from 2002 to 2015. Six expeditions were conducted with variable length of duration, covering terrestrial and fluvial routes, starting at $06 \mathrm{~h} 00$ and going until $11 \mathrm{~h} 00$, and then from $14 \mathrm{~h} 00$ until 17h30. The search for nocturnal species ran from $19 \mathrm{~h} 00$ until 22h00. Occasional and opportunistic bird registers were also recorded during our journeys, including directed visual observation and bird vocalization signals. The detected species were photographed whenever possible, to serve as evidence of occurrence. The surveyed areas are shown in Table 1. 


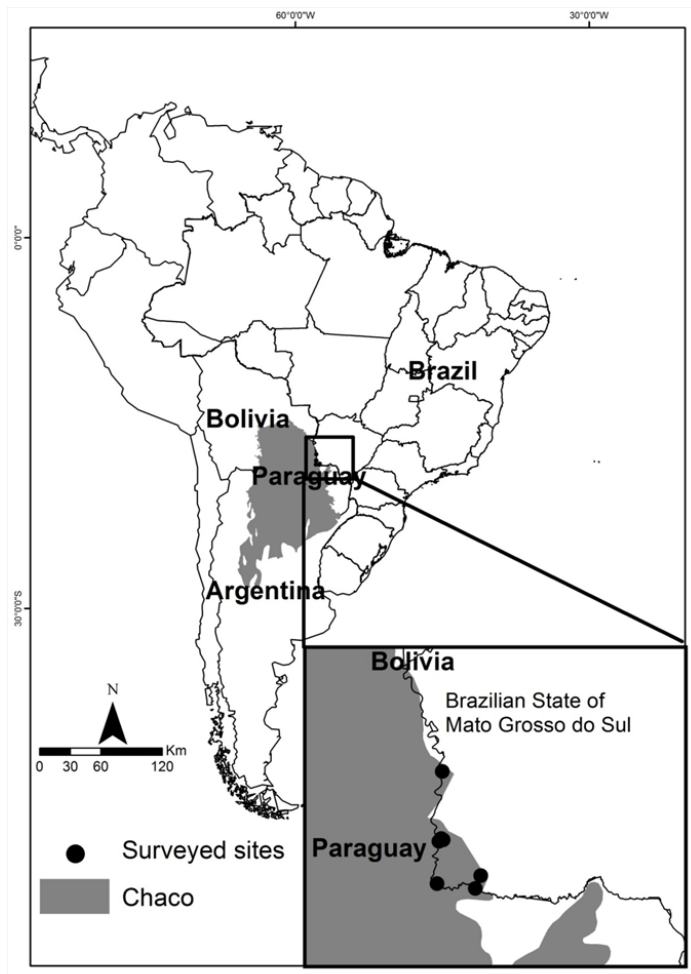

Figure I The Chaco in South America comprising the countries of Argentina, Bolivia, Paraguay and Brazil.

\section{Characteristics of the surveyed sites}

Cachoeirado apa municipal park: This Park is located at the margins of the Perdido and Apa rivers, on the border between Brazil and Paraguay. The habitats include gallery forest, seasonal forest and savanna formations (Cerrado) with influence of Chaco environment. The Park is included in the rural area, lying $85 \mathrm{~km}$ from the urban zone of Porto Murtinho.
Urban area of porto murtinho and paraguay river banks: The urban zone of the town of Porto Murtinho is located on the left bank of the Paraguay River, and despite the presence of 15,000 inhabitants, there are still some remnants of natural vegetation. Some key-trees are present: species of Prosopis, Zizyphus, Schinopsis, Aspidosperma and others.

Barranco branco station: This site is characterized by typical Chaco vegetation, including the predominance of the caranda palm Copernicia alba (common in steppe park savanna) on the left bank of the Paraguay River. Flooded fields are present along with the typical phyto physiognomies of Chaco: woody-grassy savanna, steppe scrub savanna and forested steppe savanna.

Ingazeira station: This area also presents typical Chaco vegetation such as riparian forest, deciduous forest, savanna forest; swamp sand flooded open fields with significant presence of woody-grassy savanna, steppe scrub savanna, steppe park savanna and forested steppe savanna.

Dam to control flooding and rural area: The vegetation cover of this study site is mainly formed by steppe woody-grassy savanna, steppe park savanna, forest steppe savanna and steppe scrub savanna. Besides the presence of the palm caranda Copernicia alba, other trees were noted such as Proposis spp. and Zizyphus oblongifolius.

Road BR-267/MS: Along this road and its surroundings there is vegetation cover like savanna (Cerrado), remnants of seasonal deciduous forest, and transitions to the Pantanal wetland.

Cerro pora cattle ranch: This region shows a predominance of savanna (Cerrado), seasonal deciduous forest and gallery forest. The Chaco vegetation is restricted to some transition to Cerrado. Open fields are present. The area is used for cattle ranching.

Cangalha cattle ranch: This area is also used for cattle ranching, with some vegetation cover still present, including Cerrado and flooded fields. The species names and taxonomic arrangements adopted here follow. ${ }^{14}$

Table I Surveyed area covered by field work from 2002 to 2015 throughout the Brazilian Chaco region near Porto Murtinho

\begin{tabular}{|c|c|c|c|}
\hline Surveyed sites & $\begin{array}{l}\text { Duration of the } \\
\text { survey (days) }\end{array}$ & Survey period & $\begin{array}{l}\text { Geographic } \\
\text { coordinates }\end{array}$ \\
\hline $\begin{array}{l}\text { Cachoeira do Apa Municipal } \\
\text { Park }\end{array}$ & 4 & August 2002, January 2003 & $\begin{array}{l}22^{\circ} 10^{\prime} 21.08^{\prime \prime S} \\
57^{\circ} 30^{\prime} 58.5^{\prime \prime} \mathrm{W}\end{array}$ \\
\hline Urban area of Porto Murtinho & 2-Jan & $\begin{array}{l}\text { August 2002, January 2003, February 2008, June } \\
2015 \text {, October } 2015\end{array}$ & $\begin{array}{l}21^{\circ} 4 I^{\prime} 42.0 I^{\prime \prime S} \\
57^{\circ} 52^{\prime} 42.2 " \mathrm{~W}\end{array}$ \\
\hline Margin of the Paraguay River & 2-Jan & $15-O c t$ & $\begin{array}{l}21^{\circ} 42^{\prime} 37.7 " \mathrm{~S} \\
57^{\circ} 54^{\prime} 8.7^{\prime \prime W}\end{array}$ \\
\hline Barranco Branco Station & 4 & February 2008, June 2015 & $\begin{array}{l}21^{\circ} 5^{\prime} 58.6 " \mathrm{~S} \\
57^{\circ} 50^{\prime} 37.8 " \mathrm{~W}\end{array}$ \\
\hline Ingazeira Station & 3 & February 2008, June 2015 & $\begin{array}{l}22^{\circ} 4^{\prime} 53.6^{\prime \prime S} \\
57^{\circ} 56^{\prime} 23.1 \mathrm{I} \mathrm{W}\end{array}$ \\
\hline $\begin{array}{l}\text { Dam to Control Flooding and } \\
\text { Rural Area }\end{array}$ & 2-Jan & February 2008, June 2015, October 2015 & $\begin{array}{l}21^{\circ} 4 I^{\prime} 18 . I^{\prime \prime S} \\
57^{\circ} 52^{\prime} 46.8^{8} \mathrm{~W}\end{array}$ \\
\hline Road BR- 267/MS & I & $\begin{array}{l}\text { February 2008, June } 2015 \text {, } \\
15-\text {-Oct }\end{array}$ & $\begin{array}{l}21^{\circ} 4 I^{\prime} 59.33^{\prime \prime S} \\
57^{\circ} 5 I^{\prime} 47.38 " \mathrm{~W}\end{array}$ \\
\hline Cerro Pora Cattle Ranch & 4 & Dec-15 & $\begin{array}{l}22^{\circ} 0 I^{\prime} 44.0^{\prime \prime S} \\
57^{\circ} 3 I^{\prime} 22.0^{\prime \prime} \mathrm{W}\end{array}$ \\
\hline Cangalha Cattle Ranch & 4 & Dec-15 & $\begin{array}{l}22^{\circ} 08^{\prime} 2 I^{\prime \prime S} \\
57^{\circ} 34^{\prime} 50^{\prime \prime} \mathrm{W}\end{array}$ \\
\hline
\end{tabular}




\section{Results and discussion}

Chaco or "chaku", in the Quechua language, means hunting territory, with high bird richness. The Brazilian part of the Chaco harbors different habitats for bird species, with influence of the savanna floral province (Cerrado) and the Pantanal wetland. However, the dry vegetation formation of the Chaco has its own characteristics: it is an environment that is more xeric than hydric, with some plant species like S. balansae, Aspidosperma quebrachoblanco, Caesalpinia paraguariensis, Acacia spp., Prosopis spp. and others. We registered 326 bird species during our survey. The most representative bird families were Tyrannidae (13.2\%), Thraupidae $(9.2 \%)$ and Icteridae (4.9\%). Among the non Passeriformes the families with most representatives were Accipitridae (8.3\%), Picidae (7.7\%) and Psittacidae (7.7\%). The Tyrannidae is the largest bird taxonomic family of the Neotropics, representing about $18 \%$ of the passeri forms of South America. ${ }^{15}$ This family includes frugivore and phytovore species. ${ }^{14}$ Among the Icteridae are the blackbirds, much sought after by birdwatchers. The list of the bird species encountered during our survey is summarized in Table 2. The number of bird species (326), registered in our present field survey, contributes to the ornithological knowledge of the region. Our results show that 253 species had already been registered in a previous survey. ${ }^{11}$ Comparing our study to another survey carried out in the Argentine Chaco, ${ }^{16}$ which accounted for the occurrence of 96 species living in the understory of the forest, 69 species were coincident with our present study. Comparing our results to Bolivia, 80 species out of a total of 110 species found in a Bolivian Chaco survey ${ }^{17}$ were also recorded in our present study. In connection with the Paraguay part of the Chaco, 233 bird species were registered there, ${ }^{18}$ and 194 species of those were also registered in this present study. Therefore, our survey shows the occurrence of 73 bird species which represent a new register for the Brazilian Chaco. Thus, the present survey plus the previous one for the Brazilian $\mathrm{Chaco}^{11}$ totalize 355 bird species for the region. The species Saltator multicolor deserves special reference, as it was recently found in the region, and its photographic register is documented in Wiki Aves. ${ }^{19}$ This is an endemic species living in xeric forest of the Chaco. ${ }^{7,20,21}$ Our present study also registered the occurrence of representative or endemic species of the Chaco, previously registered by other researchers, ${ }^{7,20,22,23}$ such as Rhea americana (cf. $R$. americana araneipes), Nothura boraquira, Ortalis canicollis, Phaethornis subochraceus, Nystalus striatipectus, Melanerpes cactorum, Celeus lugubris, Campephilus leucopogon, Cariama cristata, Thectocercus acuticaudatus, Aratinga nenday, Pyrrhura devillei, Myiopsitta monachus, Amazona aestiva(cf. Amazona aestiva xanthopteryx), Thamnophilus doliatus, Thamnophilus caerulescens ( $c f . T$. caerulescens paraguayensis), Campylorhamphus trochilirostris(cf. C. trochilirostris lafresnayanus), Xiphocolaptes major, Pseudoseisura unirufa, Xenopsaris a. albinucha, Suiriri suiriri, Xolmis irupero, Paroaria capitata, Saltatricula multicolor and Microspingus melanoleucus.

The birds of the Chaco exhibit very low avian endemism ${ }^{7}$ and they present very close affinities with the surrounding avifauna. ${ }^{10,24-27}$ However, the region of Porto Murtinho presents species of birds which are particular to the Chaco, ${ }^{23}$ with a close bio geographical connection with the nearby Cerrado and the Pantanal biomes. Considering the location of the Chaco and its limits to the west with the Andes, the Amazon in the north, and the Pampas and Patagonia in the south, there has been a high bio geographical interest in the region. ${ }^{20,23,28-30}$ Open habitats favor insectivore species, and the Tyrannidae are more abundant. We found $35.6 \%$ of insectivore species in our survey. They rely mostly on arthropod prey, complementing their diet with other items. ${ }^{15}$ Besides, many Tyrannidae species are austral migrants. Among the omnivores, the combination of terrestrial and hydric habitats favor the offer of invertebrate preys, amphibians and fish, depending on the annual seasonality. ${ }^{31}$ We found $32.6 \%$ of omnivore species. Our results show that the majority of the species occurrence was found in open areas $(n=130 ; 39.9 \%)$, and the other portion occurs in generalist habitats: forest, open areas and modified habitats $(n=87$; $26.7 \%)$, only in forest $(n=44 ; 13.5 \%)$, and in aquatic areas $(n=65$; $19.9 \%$ ). Some birds depend upon the presence of well preserved open habitats, including species of Furnariidae, Tyrannidae, Thraupidae, and Cardinalidae. Therefore, deforestation and conversion of natural vegetation, particularly open natural areas, is one of the major threats to be considered in conservation measures. $3,4,8,82-30$

Table 2 Surveyed area covered by field work from 2002 to 2015 throughout the Brazilian Chaco region near Porto Murtinho

\begin{tabular}{|c|c|c|c|c|c|}
\hline Taxon & English Name & Habitat & Status & Trophic guild & Surveyed Sites \\
\hline \multicolumn{6}{|l|}{ Rheiformes } \\
\hline \multicolumn{6}{|l|}{ Rheidae } \\
\hline Rhea Americana & Greater Rhea & & & ONI & $\mathrm{I}, 2,5,7,8$ \\
\hline \multicolumn{6}{|l|}{ Tinamiformes } \\
\hline Crypturellus Undulatus & Undulated Tinamou & FO & & ONI & $\mathrm{I}, 4,5,8,9$ \\
\hline Crypturellus Parvirostris & Small-Billed Tinamou & $\mathrm{OPCe}$ & & ONI & $\mathrm{I}, 2,5,6,8$ \\
\hline Crypturellus Tataupa & Tataupa Tinamou & $\mathrm{FO}$ & & $\mathrm{ONI}$ & $1,4,5,8,9$ \\
\hline Rhynchotus Rufescens & Red-Winged Tinamou & $\mathrm{OP} \mathrm{Ce}$ & & ONI & 7,8 \\
\hline Nothura Maculosa & Spotted Nothura & $\mathrm{OP} \mathrm{Ce}$ & & ONI & $\mathrm{I}, 7,8,9$ \\
\hline \multicolumn{6}{|l|}{ Anseriformes } \\
\hline \multicolumn{6}{|l|}{ Anhimidae } \\
\hline Chauna Torquata & Southern Screamer & $A$ & & ONI & $\mathrm{I}, 2,6,7$ \\
\hline
\end{tabular}


Table Continued....

\begin{tabular}{|c|c|c|c|c|c|}
\hline Taxon & English Name & Habitat & Status & Trophic guild & Surveyed Sites \\
\hline \multicolumn{6}{|l|}{ Anatidae } \\
\hline Dendrocygna Autumnalis & Black-Bellied Whistling-Duck & $A$ & $\mathrm{R}$ & ONI & $\mathrm{I}, 2,5,7,8$ \\
\hline Cairina Moschata & Muscovy Duck & $A$ & $\mathrm{R}$ & ONI & $1,2,5,6,7,9,9$ \\
\hline Callonetta Leucophrys & Ringed Teal & $A$ & AM & ONI & 7 \\
\hline Amazonetta Brasiliensis & Brazilian Teal & A & $\mathrm{R}$ & ONI & 2,5 \\
\hline Nomonyx Dominicus & Masked Duck & $A$ & $\mathrm{R}$ & ONI & 7 \\
\hline \multicolumn{6}{|l|}{ Galliformes } \\
\hline \multicolumn{6}{|l|}{ Cracidae } \\
\hline Penelope Superciliaris & Rusty-Margined Guan & FO & & FRU & $1,8,9$ \\
\hline Aburria Cumanensis & Blue-Throated Piping-Guan & FO & & FRU & $1,2,3,4,5,6,9$ \\
\hline Ortalis Canicollis & Chaco Chachalaca & $\mathrm{FO}, \mathrm{OP} \mathrm{Ce}, \mathrm{OP} \mathrm{Ch}$ & & FRU & I,2,3,4,5,6,7,8,9 \\
\hline Crax Fasciolata & Bare-Faced Curassow & FO & & FRU & $1,4,5$ \\
\hline \multicolumn{6}{|l|}{ Podicipediformes } \\
\hline \multicolumn{6}{|l|}{ Podicipedidae } \\
\hline Tachybaptus Dominicus & Least Grebe & $A$ & $\mathrm{R}$ & ONI & 5,7 \\
\hline \multicolumn{6}{|l|}{ Ciconiiformes } \\
\hline \multicolumn{6}{|l|}{ Ciconiidae } \\
\hline Ciconia Maguari & Maguari Stork & $A$ & ICA & PIS/CAR & 5,7 \\
\hline Jabiru Mycteria & Jabiru & $A$ & ICA & PIS/CAR & $2,5,6,7$ \\
\hline Mycteria Americana & Wood Stork & $A$ & ICA & PIS & $4,5,6,7$ \\
\hline \multicolumn{6}{|l|}{ Suliformes } \\
\hline \multicolumn{6}{|l|}{ Phalacrocoracidae } \\
\hline Nannopterum Brasilianus & Neotropic Cormorant & $A$ & $\mathrm{R}$ & PIS & I,3,4,5,6 \\
\hline \multicolumn{6}{|l|}{ Anhingidae } \\
\hline Anhinga Anhinga & Anhinga & $A$ & & PIS & $\mathrm{I}, 3,4,5,6,7$ \\
\hline \multicolumn{6}{|l|}{ Pelecaniformes } \\
\hline \multicolumn{6}{|l|}{ Ardeidae } \\
\hline Tigrisoma Lineatum & Rufescent Tiger-Heron & $A$ & & PIS/CAR & $1,3,5,6,7,8,9$ \\
\hline Cochlearius cochlearius & Boat-Billed Heron & $A$ & & PIS/CAR & 1 \\
\hline Nycticorax Nycticorax & Black-Crowned Night-Heron & $A$ & & ONI & $\mathrm{I}, 2,3,5$ \\
\hline Butorides Striata & Striated Heron & $A$ & ICA & ONI & $\mathrm{I}, 2,3,4,5,6,7$ \\
\hline Bubulcus Ibis & Cattle Egret & AA & ICA & INS & $2,5,7,8,9$ \\
\hline Ardea Cocoi & Cocoi Heron & A & & PIS/CAR & I,3,4,5,7 \\
\hline Ardea Alba & Great Egret & $A$ & ICA & PIS/CAR & $\mathrm{I}, 2,3,4,5,6,7,8,9$ \\
\hline Syrigma Sibilatrix & Whistling Heron & $\mathrm{A}, \mathrm{OP} \mathrm{Ce}, \mathrm{OP} \mathrm{Ch}$ & & ONI & $\mathrm{I}, 2,4,5,7,8$ \\
\hline Pilherodius Pileatus & Capped Heron & $A$ & & PIS/CAR & $1,5,7$ \\
\hline Egretta Thula & Snowy Egret & A & ICA & $\mathrm{PIS} / \mathrm{CAR}$ & $\mathrm{I}, 2,3,4,5,6,7$ \\
\hline \multicolumn{6}{|l|}{ Threskiornithidae } \\
\hline Plegadis Chihi & White-Faced lbis & $A$ & ICA & ONI & 7 \\
\hline Mesembrinibis Cayennensis & Green Ibis & $\mathrm{A}, \mathrm{FO}$ & $\mathrm{R}$ & ONI & $\mathrm{I}, 5$ \\
\hline
\end{tabular}


Table Continued...

\begin{tabular}{|c|c|c|c|c|c|}
\hline Taxon & English Name & Habitat & Status & Trophic guild & Surveyed Sites \\
\hline Phimosus Infuscatus & Bare-Faced lbis & $A$ & $\mathrm{R}$ & ONI & $2,5,6,7,8$ \\
\hline Theristicus Caerulescens & Plumbeous lbis & $\mathrm{A}, \mathrm{FO}$ & & ONI & I, 2,3,4,5,6,7,8,9 \\
\hline Theristicus Caudatus & Buff-Necked lbis & $\mathrm{A}, \mathrm{OP} \mathrm{Ce}, \mathrm{OP} \mathrm{Ch}$ & & ONI & I,2,5,7,8 \\
\hline Platalea Ajaja & Roseate Spoonbill & $A$ & ICA & ONI & $\mathrm{I}, 2,5,7$ \\
\hline \multicolumn{6}{|l|}{ Cathartiformes } \\
\hline \multicolumn{6}{|l|}{ Cathartidae } \\
\hline Cathartes Aura & Turkey Vulture & $\mathrm{FO}, \mathrm{OP} \mathrm{Ce}, \mathrm{OP} \mathrm{Ch}$ & AM & DET & $\mathrm{I}, 2,4,5,7,8,9$ \\
\hline Cathartes Burrovianus & Lesser Yellow-Headed Vulture & $\mathrm{FO}, \mathrm{OP} \mathrm{Ce}, \mathrm{OP} \mathrm{Ch}$ & & DET & $\mathrm{I}, 2,3,4,5,6,7,8,9$ \\
\hline Coragyps Atratus & Black Vulture & $\mathrm{AA}, \mathrm{OP} \mathrm{Ce}, \mathrm{OP} \mathrm{Ch}$ & & DET & $\mathrm{I}, 2,3,4,5,6,7,8,9$ \\
\hline Sarcoramphus Papa & King Vulture & $\mathrm{OPCe}$ & & DET & $\mathrm{I}, 8$ \\
\hline \multicolumn{6}{|l|}{ Accipitriformes } \\
\hline \multicolumn{6}{|l|}{ Pandionidae } \\
\hline Pandion Haliaetus & Osprey & $A$ & S & PIS & $1,3,4$ \\
\hline \multicolumn{6}{|l|}{ Accipitridae } \\
\hline Leptodon Cayanensis & Gray-Headed Kite & $\mathrm{FO}$ & & CAR & I \\
\hline Chondrohierax Uncinatus & Hook-Billed Kite & FO & & CAR & 5,7 \\
\hline Gampsonyx Swainsonii & Pearl Kite & $\mathrm{FO}, \mathrm{OP} \mathrm{Ce}, \mathrm{OP} \mathrm{Ch}$ & & CAR & $\mathrm{I}, 2$ \\
\hline Elanus Leucurus & White-Tailed Kite & $\mathrm{OP} \mathrm{Ce}, \mathrm{OP} \mathrm{Ch}$ & & CAR & $2,5,7,8$ \\
\hline Accipiter Striatus & Sharp-Shinned Hawk & $\mathrm{FO}$ & & CAR & 5 \\
\hline Ictinia Plumbea & Plumbeous Kite & OP Ce, OP Ch & $\mathrm{R}$ & CAR/INS & I,4,7,8,9 \\
\hline Busarellus Nigricollis & Black-Collared Hawk & $A$ & & PIS/CAR & $\mathrm{I}, 2,3,4,5,6,7$ \\
\hline Rostrhamus Sociabilis & Snail Kite & A & $\mathrm{R}$ & MAL & $\mathrm{I}, 2,5,6,7$ \\
\hline Geranospiza Caerulescens & Crane Hawk & $\mathrm{FO}, \mathrm{OP} \mathrm{Ce}, \mathrm{OP} \mathrm{Ch}$ & & CAR & $\mathrm{I}, 2,4,5,7$ \\
\hline Heterospizias Meridionalis & Savanna Hawk & $\mathrm{OP} \mathrm{Ce}, \mathrm{OP} \mathrm{Ch}$ & & CAR & $1,2,3,4,5,6,7,8,9$ \\
\hline Urubitinga Urubitinga & Great Black Hawk & $\mathrm{OP} \mathrm{Ce}, \mathrm{OP} \mathrm{Ch}$ & & CAR & I,2,4,5,7 \\
\hline Urubitinga Coronata & Crowned Eagle & $\mathrm{OP} \mathrm{Ce}, \mathrm{OP} \mathrm{Ch}$ & & CAR & 5 \\
\hline Rupornis Magnirostris & Roadside Hawk & $\mathrm{OP} \mathrm{Ce}, \mathrm{OP} \mathrm{Ch}$ & & CAR & $\mathrm{I}, 2,4,5,6,7,8,9$ \\
\hline Geranoaetus Albicaudatus & White-Tailed Hawk & $\mathrm{OPCe}$ & & CAR & I,2,7,8,9 \\
\hline \multicolumn{6}{|l|}{ Gruiformes } \\
\hline \multicolumn{6}{|l|}{ Aramidae } \\
\hline Aramus Guarauna & Limpkin & $A$ & $\mathrm{R}$ & MAL & $1,2,4,5,6,7,9$ \\
\hline \multicolumn{6}{|l|}{ Rallidae } \\
\hline Aramides Ypecaha & Giant Wood-Rail & A & & ONI & I,2,4,5,7,8,9 \\
\hline Aramides Cajaneus & Gray-Necked Wood-Rail & $A$ & & ONI & $1,2,5$ \\
\hline Laterallus Melanophaius & Rufous-Sided Crake & $A$ & & ONI & 5 \\
\hline Mustelirallus Albicollis & Ash-Throated Crake & $A$ & & ONI & $2,5,8$ \\
\hline Pardirallus Maculatus & Spotted Rail & A & ICA & ONI & 5,7 \\
\hline Pardirallus Nigricans & Blackish Rail & A & ICA & ONI & 5,7 \\
\hline Gallinula Galeata & Common Gallinule & A & ICA & ONI & $2,5,7$ \\
\hline Porphyrio Martinicus & Purple Gallinule & A & ICA & ONI & I, $2,5,6,7$ \\
\hline
\end{tabular}


Table Continued....

\begin{tabular}{|c|c|c|c|c|c|}
\hline Taxon & English Name & Habitat & Status & Trophic guild & Surveyed Sites \\
\hline \multicolumn{6}{|l|}{ Heliornithidae } \\
\hline Heliornis Fulica & Sungrebe & A & ICA & ONI & $\mathrm{I}, 4$ \\
\hline \multicolumn{6}{|l|}{ Charadriiformes } \\
\hline \multicolumn{6}{|l|}{ Charadriidae } \\
\hline Vanellus Chilensis & Southern Lapwing & AA, OP Ce, OP Ch & & ONI & $\mathrm{I}, 2,3,4,5,6,7,8,9$ \\
\hline Charadrius Collaris & Collared Plover & $A$ & ICA & ONI & 4,5 \\
\hline \multicolumn{6}{|l|}{ Recurvirostridae } \\
\hline Himantopus Melanurus & White-Backed Stilt & A & $\mathrm{R}$ & ONI & $\mathrm{I}, 5,6,7$ \\
\hline \multicolumn{6}{|l|}{ Scolopacidae } \\
\hline Gallinago Paraguaiae & South American Snipe & $A$ & ICA & ONI & 7 \\
\hline Bartramia Longicauda & Upland Sandpiper & OP Ce, OP Ch & $\mathrm{S}$ & ONI & 7 \\
\hline Tringa Solitaria & Solitary Sandpiper & $A$ & $\mathrm{~S}$ & ONI & $\mathrm{I}, 5,7,8$ \\
\hline Tringa Melanoleuca & Greater Yellowlegs & A & $S$ & ONI & 7 \\
\hline Tringa Flavipes & Lesser Yellowlegs & A & $\mathrm{S}$ & ONI & $\mathrm{I}, 7$ \\
\hline Calidris Fuscicollis & White-Rumped Sandpiper & $A$ & $\mathrm{~S}$ & ONI & 7 \\
\hline Calidris Melanotos & Pectoral Sandpiper & $A$ & $\mathrm{~S}$ & ONI & 7 \\
\hline Calidris Himantopus & Stilt Sandpiper & A & $\mathrm{S}$ & ONI & 7 \\
\hline \multicolumn{6}{|l|}{ Jacanidae } \\
\hline Jacana Jacana & Wattled Jacana & $A$ & & ONI & $1,2,4,5,6,7,8,9$ \\
\hline Sternula Superciliaris & Yellow-Billed Tern & A & $\mathrm{R}$ & PIS & $\mathrm{I}, 2,3,5$ \\
\hline Phaetusa Simplex & Large-Billed Tern & A & $\mathrm{R}$ & PIS & $\mathrm{I}, 2,3,5$ \\
\hline \multicolumn{6}{|l|}{ Rynchopidae } \\
\hline Rynchops Niger & Black Skimmer & A & $\mathrm{R}$ & PIS & $\mathrm{I}, 3,7$ \\
\hline \multicolumn{6}{|l|}{ Columbiformes } \\
\hline \multicolumn{6}{|l|}{ Columbidae } \\
\hline Columbina Minuta & Plain-Breasted Ground-Dove & OP Ce, OP Ch & & ONI & $5,6,9$ \\
\hline Columbina Talpacoti & Ruddy Ground-Dove & OP Ce, OP Ch & $\mathrm{R}$ & ONI & $\mathrm{I}, 2,3,4,5,6,7,8,9$ \\
\hline Columbina Squammata & Scaled Dove & $\mathrm{OP} \mathrm{Ce}, \mathrm{OP} \mathrm{Ch}$ & & ONI & $\mathrm{I}, 2,4,5,6,8,9$ \\
\hline Columbina Picui & Picui Ground-Dove & $\mathrm{OP} \mathrm{Ce}, \mathrm{OP} \mathrm{Ch}$ & $\mathrm{R}$ & ONI & $\mathrm{I}, 2,3,4,5,6,7,8,9$ \\
\hline Claravis Pretiosa & Blue Ground-Dove & $\mathrm{FO}, \mathrm{OP} \mathrm{Ce}, \mathrm{OP} \mathrm{Ch}$ & $\mathrm{R}$ & ONI & $\mathrm{I}, 4,5,8$ \\
\hline Columba Livia & Rock Pigeon & $\mathrm{AA}$ & & ONI & 2 \\
\hline Patagioenas Picazuro & Picazuro Pigeon & AA, FO, OP Ce, OP Ch & $\mathrm{R}$ & ONI & I,2,4,5,6,7,8,9 \\
\hline Patagioenas Cayennensis & Pale-Vented Pigeon & $\mathrm{AA}, \mathrm{FO}, \mathrm{OP} \mathrm{Ce}, \mathrm{OP} \mathrm{Ch}$ & $\mathrm{R}$ & ONI & $\mathrm{I}, 2,4,5,7,8,9$ \\
\hline Zenaida Auriculata & Eared Dove & $\mathrm{AA}, \mathrm{OP} \mathrm{Ce}, \mathrm{OP} \mathrm{Ch}$ & $\mathrm{R}$ & ONI & $\mathrm{I}, 2,3,4,5,6,7,8,9$ \\
\hline Leptotila Verreauxi & White-Tipped Dove & FO, OP Ce, OP Ch & & ONI & I, $2,4,5,6,8,9$ \\
\hline Leptotila Rufaxilla & Gray-Fronted Dove & $\mathrm{FO}, \mathrm{OP} \mathrm{Ce}$ & & ONI & $1,8,9$ \\
\hline \multicolumn{6}{|l|}{ Cuculiformes } \\
\hline Cuculidae & & & & & \\
\hline
\end{tabular}


Table Continued....

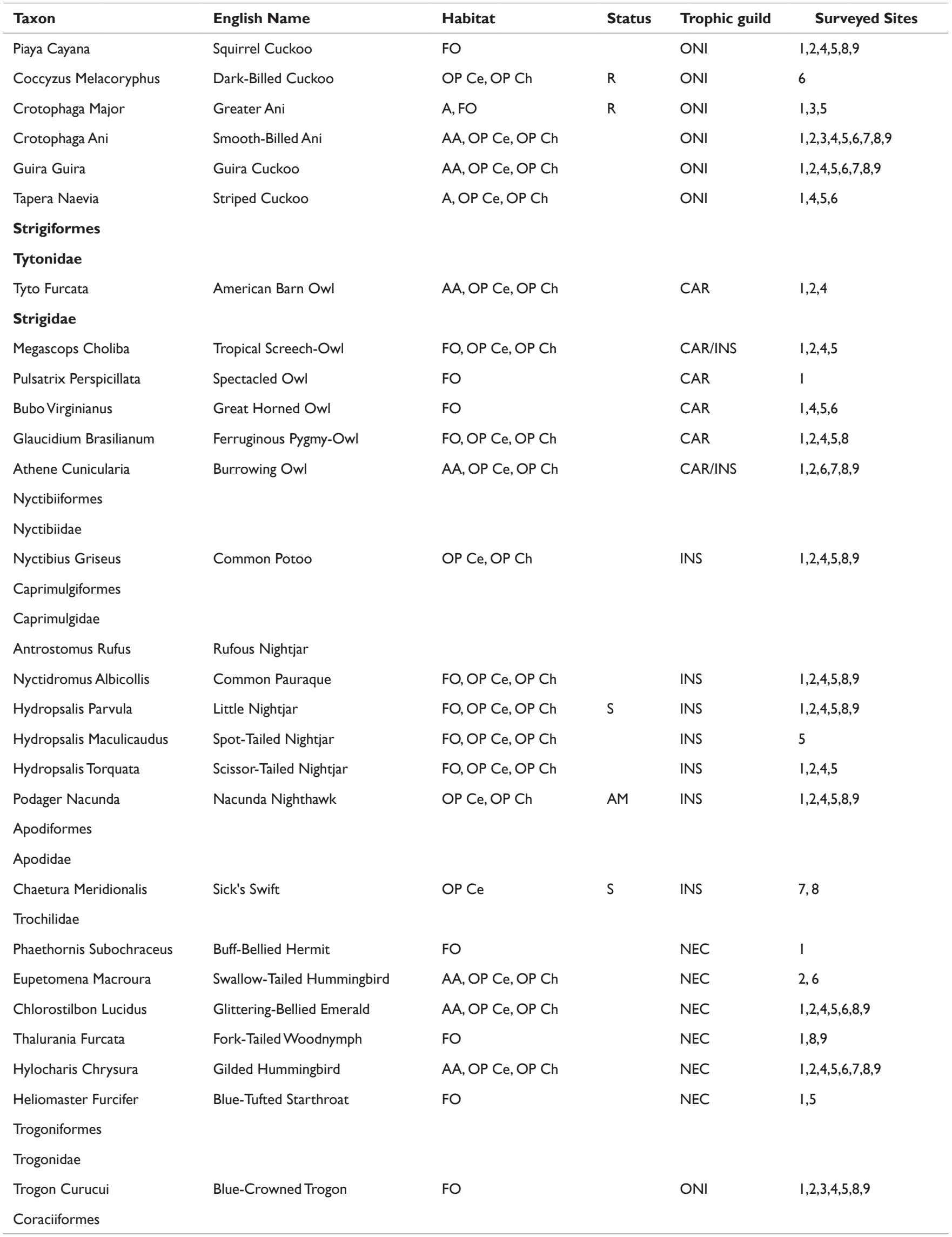


Table Continued....

\begin{tabular}{|c|c|c|c|c|c|}
\hline Taxon & English Name & Habitat & Status & Trophic guild & Surveyed Sites \\
\hline \multicolumn{6}{|l|}{ Alcedinidae } \\
\hline Megaceryle Torquata & Ringed Kingfisher & A & & PIS & $\mathrm{I}, 2,3,4,5,7,8$ \\
\hline Chloroceryle Amazona & Amazon Kingfisher & $A$ & & PIS & $1,3,5$ \\
\hline Chloroceryle Aenea & American Pygmy Kingfisher & $A$ & & PIS & I \\
\hline Chloroceryle Americana & Green Kingfisher & A & & PIS & $1,3,5$ \\
\hline Chloroceryle Inda & Green-And-Rufous Kingfisher & $A$ & & PIS & \\
\hline Momotidae & & & & & I \\
\hline Momotus Momota & Amazonian Motmot & FO & & ONI & $\mathrm{I}, 2,3,4,5,7,8$ \\
\hline \multicolumn{6}{|l|}{ Galbuliformes } \\
\hline \multicolumn{6}{|l|}{ Galbulidae } \\
\hline Galbula Ruficauda & Rufous-Tailed Jacamar & FO & & INS & \\
\hline Bucconidae & & & & & 1 \\
\hline Nystalus Chacuru & White-Eared Puffbird & $\mathrm{OPCe}$ & & ONI & 8 \\
\hline Nystalus Striatipectus & Chaco Puffbird & OP Ce, OP Ch & & ONI & I,2,4,5,8,9 \\
\hline \multicolumn{6}{|l|}{ Piciformes } \\
\hline \multicolumn{6}{|l|}{ Ramphastidae } \\
\hline Ramphastos Toco & Toco Toucan & $\mathrm{AA}, \mathrm{FO}, \mathrm{OP} \mathrm{Ce}, \mathrm{OP} \mathrm{Ch}$ & & ONI/FRU & $\mathrm{I}, 2,3,4,5,6,78,9$ \\
\hline Pteroglossus Castanotis & Chestnut-Eared Aracari & FO & & ONI/FRU & $\mathrm{I}, 2,4,5,8,9$ \\
\hline \multicolumn{6}{|l|}{ Picidae } \\
\hline Picumnus Cirratus & White-Barred Piculet & OP Ce, OP Ch & & INS & $1,4,5$ \\
\hline Picumnus Albosquamatus & White-Wedged Piculet & OP Ce, OP Ch & & INS & $1,3,5$ \\
\hline Melanerpes Candidus & White Woodpecker & $\mathrm{AA}, \mathrm{OP} \mathrm{Ce}, \mathrm{OP} \mathrm{Ch}$ & & INS & $\mathrm{I}, 2,4,5,7,8,9$ \\
\hline Melanerpes Cactorum & White-Fronted Woodpecker & $\mathrm{OPCh}$ & & INS & 5 \\
\hline Veniliornis Passerinus & Little Woodpecker & $\mathrm{FO}, \mathrm{OP} \mathrm{Ce}, \mathrm{OP} \mathrm{Ch}$ & & INS & $1,2,4,5,8,9$ \\
\hline Veniliornis Mixtus & Checkered Woodpecker & OP Ce, OP Ch & & INS & $2,4,5,6$ \\
\hline Piculus Chrysochloros & Golden-Green Woodpecker & FO & & INS & $\mathrm{I}, 4,5,6,8,9$ \\
\hline Colaptes Melanochloros & Green-Barred Woodpecker & $\mathrm{AA}, \mathrm{FO}, \mathrm{OP} \mathrm{Ce}, \mathrm{OP} \mathrm{Ch}$ & & INS & $\mathrm{I}, 2,4,5,6,7,8,9$ \\
\hline Colaptes Campestris & Campo Flicker & $\mathrm{AA}, \mathrm{OP} \mathrm{Ce}, \mathrm{OP} \mathrm{Ch}$ & & INS & $1,2,4,5,6,7,8,9$ \\
\hline Celeus Lugubris & Pale-Crested Woodpecker & FO & & INS & I,3,4,5,8,9 \\
\hline Dryocopus Lineatus & Lineated Woodpecker & FO & & INS & $1,8,9$ \\
\hline Campephilus Melanoleucos & Crimson-Crested Woodpecker & $\mathrm{FO}, \mathrm{OP} \mathrm{Ce}, \mathrm{OP} \mathrm{Ch}$ & & INS & $\mathrm{I}, 2,4,5,8,9$ \\
\hline Campephilus Leucopogon & Cream-Backed Woodpecker & $\mathrm{FO}, \mathrm{OP} \mathrm{Ch}$ & & INS & 5 \\
\hline \multicolumn{6}{|l|}{ Cariamiformes } \\
\hline \multicolumn{6}{|l|}{ Cariamidae } \\
\hline Cariama Cristata & Red-Legged Seriema & OP Ce, OP Ch & & ONI & $\mathrm{I}, 2,4,5,6,7,8,9$ \\
\hline \multicolumn{6}{|l|}{ Falconiformes } \\
\hline \multicolumn{6}{|l|}{ Falconidae } \\
\hline Caracara Plancus & Southern Caracara & OP Ce, OP Ch & & ONI & $\mathrm{I}, 2,3,4,5,6,7,8,9$ \\
\hline Milvago Chimachima & Yellow-Headed Caracara & OP Ce, OP Ch & & ONI & $\mathrm{I}, 2,4,5,6,7$ \\
\hline Herpetotheres Cachinnans & Laughing Falcon & OP Ce, OP Ch & & CAR & I,2,4,5,6,7,8,9 \\
\hline
\end{tabular}


Table Continued....

\begin{tabular}{|c|c|c|c|c|c|}
\hline Taxon & English Name & Habitat & Status & Trophic guild & Surveyed Sites \\
\hline Micrastur Semitorquatus & Collared Forest-Falcon & FO & & CAR & $\mathrm{I}, 4$ \\
\hline Falco Sparverius & American Kestrel & OP Ce, OP Ch & $\mathrm{R}$ & CAR & $\mathrm{I}, 2,4,5,6,7,8,9$ \\
\hline Falco Rufigularis & Bat Falcon & OP Ce, OP Ch & & CAR & 1 \\
\hline Falco Femoralis & Aplomado Falcon & & ICA & CAR & $1,2,4,5,7,8,9$ \\
\hline \multicolumn{6}{|l|}{ Psittaciformes } \\
\hline \multicolumn{6}{|l|}{ Psittacidae } \\
\hline Anodorhynchus Hyacinthinus & Hyacinth Macaw & $\mathrm{FO}, \mathrm{OP} \mathrm{Ce}, \mathrm{OP} \mathrm{Ch}$ & & FRU & $\mathrm{I}, 7$ \\
\hline Ara Chloropterus & Red-And-Green Macaw & OP Ce, OP Ch & & FRU & $\mathrm{I}, 2,4,5,6,7,8,9$ \\
\hline Primolius Auricollis & Yellow-Collared Macaw & $\mathrm{FO}, \mathrm{OP} \mathrm{Ce}, \mathrm{OP} \mathrm{Ch}$ & & FRU & $\mathrm{I}, 4,5,7$ \\
\hline Thectocercus Acuticaudatus & Blue-Crowned Parakeet & $\mathrm{FO}, \mathrm{OP} \mathrm{Ce}, \mathrm{OP} \mathrm{Ch}$ & & FRU & $1,2,4,5$ \\
\hline Psittacara Leucophthalmus & White-Eyed Parakeet & $\mathrm{FO}, \mathrm{OP} \mathrm{Ce}, \mathrm{OP} \mathrm{Ch}$ & & FRU & $1,2,4,5,6,8,9$ \\
\hline Aratinga Nenday & Nanday Parakeet & $\mathrm{OPCe}, \mathrm{OP} \mathrm{Ch}$ & & FRU & $\mathrm{I}, 2,3,4,5,7,8,9$ \\
\hline Eupsittula Aurea & Peach-Fronted Parakeet & OP Ce, OP Ch & & FRU & 8,9 \\
\hline Pyrrhura Devillei & Blaze-Winged Parakeet & $\mathrm{FO}, \mathrm{OP} \mathrm{Ce}, \mathrm{OP} \mathrm{Ch}$ & & FRU & $\mathrm{I}, 4,5,8,9$ \\
\hline Myiopsitta Monachus & Monk Parakeet & $\mathrm{AA}, \mathrm{FO}, \mathrm{OP} \mathrm{Ce}, \mathrm{OP} \mathrm{Ch}$ & & FRU & I,2,3,4,5,6,7,8,9 \\
\hline Forpus Xanthopterygius & Blue-Winged Parrotlet & $\mathrm{OPCe}$ & & FRU & 8,9 \\
\hline Brotogeris Chiriri & Yellow-Chevroned Parakeet & $\mathrm{AA}, \mathrm{OP} \mathrm{Ce}, \mathrm{OP} \mathrm{Ch}$ & & FRU & I,2,3,4,5,6,7,8,9 \\
\hline Pionus Maximiliani & Scaly-Headed Parrot & $\mathrm{FO}, \mathrm{OP} \mathrm{Ce}, \mathrm{OP} \mathrm{Ch}$ & & FRU & $\mathrm{I}, 2,3,4,5,6,7,8,9$ \\
\hline Amazona Aestiva & Turquoise-Fronted Parrot & $\mathrm{FO}, \mathrm{OP} \mathrm{Ce}, \mathrm{OP} \mathrm{Ch}$ & & FRU & $1,2,4,5,6,8,9$ \\
\hline \multicolumn{6}{|l|}{ Passeriformes } \\
\hline \multicolumn{6}{|l|}{ Thamnophilidae } \\
\hline Formicivora Rufa & Rusty-Backed Antwren & OP Ce, OP Ch & & INS & $1,2,8,9$ \\
\hline Thamnophilus Doliatus & Barred Antshrike & $\mathrm{FO}, \mathrm{OP} \mathrm{Ce}, \mathrm{OP} \mathrm{Ch}$ & & INS & $\mathrm{I}, 2,3,4,5,6,8,9$ \\
\hline Thamnophilus Caerulescens & Variable Antshrike & $\mathrm{FO}, \mathrm{OP} \mathrm{Ce}, \mathrm{OP} \mathrm{Ch}$ & & INS & $\mathrm{I}, 4,5,8,9$ \\
\hline Taraba Major & Great Antshrike & $\mathrm{FO}, \mathrm{OP} \mathrm{Ce}, \mathrm{OP} \mathrm{Ch}$ & & INS & $\mathrm{I}, 2,3,4,5,6,8,9$ \\
\hline Cercomacra Melanaria & Mato Grosso Antbird & FO & & INS & 1,5 \\
\hline \multicolumn{6}{|l|}{ Dendrocolaptidae } \\
\hline Sittasomus Griseicapillus & Olivaceous Woodcreeper & $\mathrm{FO}, \mathrm{OP} \mathrm{Ce}, \mathrm{OP} \mathrm{Ch}$ & & INS & $\mathrm{I}, 2,3,4,5,6,8,9$ \\
\hline $\begin{array}{l}\text { Campylorhamphus } \\
\text { Trochilirostris }\end{array}$ & Red-Billed Scythebill & FO & & INS & $\mathrm{I}, 4,5$ \\
\hline Lepidocolaptes Angustirostris & Narrow-Billed Woodcreeper & OP Ce, OP Ch & & INS & $\mathrm{I}, 2,4,5,6,7,8,9$ \\
\hline Dendrocolaptes Platyrostris & Planalto Woodcreeper & FO & & INS & 8,9 \\
\hline Xiphocolaptes Major & Great Rufous Woodcreeper & $\mathrm{FO}, \mathrm{OP} \mathrm{Ce}, \mathrm{OP} \mathrm{Ch}$ & & INS & I,2,4,5,8,9 \\
\hline \multicolumn{6}{|l|}{ Furnariidae } \\
\hline Furnarius Leucopus & Pale-Legged Hornero & FO & & ONI & 1 \\
\hline Furnarius Rufus & Rufous Hornero & $\mathrm{AA}, \mathrm{FO}, \mathrm{OP} \mathrm{Ce}, \mathrm{OP} \mathrm{Ch}$ & & ONI & $\mathrm{I}, 2,3,4,5,6,7,8,9$ \\
\hline Pseudoseisura Unirufa & Rufous Cacholote & $\mathrm{FO}, \mathrm{OP} \mathrm{Ce}, \mathrm{OP} \mathrm{Ch}$ & & INS & $\mathrm{I}, 2,4,5$ \\
\hline Phacellodomus Rufifrons & Rufous-Fronted Thornbird & $\mathrm{OPCe}, \mathrm{OP} \mathrm{Ch}$ & & INS & $1,2,4,5,6,8,9$ \\
\hline Phacellodomus Ruber & Greater Thornbird & $\mathrm{OPCe}, \mathrm{OP} \mathrm{Ch}$ & & INS & I,2,4,5,8,9 \\
\hline Anumbius Annumbi & Firewood-Gatherer & OP Ce, OP Ch & & INS & 5,7 \\
\hline
\end{tabular}


Table Continued....

\begin{tabular}{|c|c|c|c|c|c|}
\hline Taxon & English Name & Habitat & Status & Trophic guild & Surveyed Sites \\
\hline $\begin{array}{l}\text { Schoeniophylax } \\
\text { Phryganophilus }\end{array}$ & Chotoy Spinetail & $\mathrm{OP} \mathrm{Ce}, \mathrm{OP} \mathrm{Ch}$ & & INS & $\mathrm{I}, 2,4,5,7,8$ \\
\hline Certhiaxis Cinnamomeus & Yellow-Chinned Spinetail & $A$ & & INS & $1,2,3,4,5,7,8,9$ \\
\hline Synallaxis Frontalis & Sooty-Fronted Spinetail & $\mathrm{FO}, \mathrm{OP} \mathrm{Ce}, \mathrm{OP} \mathrm{Ch}$ & & INS & $\mathrm{I}, 2,6,8,9$ \\
\hline Synallaxis Hypospodia & Cinereous-Breasted Spinetail & $A$ & & INS & $5,8,9$ \\
\hline Synallaxis Albilora & White-Lored Spinetail & FO & & INS & $\mathrm{I}, 3,4,5,8,9$ \\
\hline Cranioleuca Vulpina & Rusty-Backed Spinetail & $\mathrm{A}, \mathrm{FO}$ & & INS & $1,4,5$ \\
\hline \multicolumn{6}{|l|}{ Pipridae } \\
\hline Pipra Fasciicauda & Band-Tailed Manakin & FO & & FRU & I \\
\hline \multicolumn{6}{|l|}{ Tityridae } \\
\hline Tityra Inquisitor & Black-Crowned Tityra & $\mathrm{FO}, \mathrm{OP} \mathrm{Ce}, \mathrm{OP} \mathrm{Ch}$ & & ONI & I,4,5,8,9 \\
\hline Tityra Cayana & Black-Tailed Tityra & $\mathrm{FO}, \mathrm{OP} \mathrm{Ce}, \mathrm{OP} \mathrm{Ch}$ & & ONI & $\mathrm{I}, 4,5,8,9$ \\
\hline Pachyramphus Viridis & Green-Backed Becard & $\mathrm{FO}, \mathrm{OP} \mathrm{Ce}, \mathrm{OP} \mathrm{Ch}$ & & INS & I,4,5,8,9 \\
\hline Pachyramphus Polychopterus & White-Winged Becard & $\mathrm{FO}, \mathrm{OP} \mathrm{Ce}, \mathrm{OP} \mathrm{Ch}$ & AM & INS & $1,2,4,5,8,9$ \\
\hline Pachyramphus Validus & Crested Becard & $\mathrm{FO}, \mathrm{OP} \mathrm{Ce}$ & AM & INS & 8,9 \\
\hline Xenopsaris Albinucha & White-Naped Xenopsaris & $\mathrm{OPCe}, \mathrm{OP} \mathrm{Ch}$ & & INS & 7 \\
\hline \multicolumn{6}{|l|}{ Platyrinchidae } \\
\hline Platyrinchus Mystaceus & White-Throated Spadebill & FO & & INS & $1,8,9$ \\
\hline \multicolumn{6}{|l|}{ Rhynchocyclidae } \\
\hline Leptopogon Amaurocephalus & Sepia-Capped Flycatcher & FO & AM & INS & $\mathrm{I}, 4,5,8,9$ \\
\hline Tolmomyias Sulphurescens & Yellow-Olive Flycatcher & FO & & INS & $1,5,8,9$ \\
\hline Todirostrum Cinereum & Common Tody-Flycatcher & $\mathrm{AA}, \mathrm{FO}, \mathrm{OP} \mathrm{Ce}$ & & INS & $1,2,8,9$ \\
\hline $\begin{array}{l}\text { Hemitriccus } \\
\text { Margaritaceiventer }\end{array}$ & Pearly-Vented Tody-Tyrant & OP Ce, OP Ch & & INS & $\mathrm{I}, 2,4,5,6,8,9$ \\
\hline \multicolumn{6}{|l|}{ Tyrannidae } \\
\hline Inezia Inornata & Plain Tyrannulet & OP Ce, OP Ch & AM & INS & $\mathrm{I}, 4,5,8,9$ \\
\hline Euscarthmus Meloryphus & Tawny-Crowned Pygmy-Tyrant & $\mathrm{OPCe}, \mathrm{OP} \mathrm{Ch}$ & AM & INS & I,4,5,8,9 \\
\hline Camptostoma Obsoletum & Southern Beardless-Tyrannulet & $\mathrm{FO}, \mathrm{OP} \mathrm{Ce}, \mathrm{OP} \mathrm{Ch}$ & & INS & $1,2,3,4,5,6,8,9$ \\
\hline Elaenia Flavogaster & Yellow-Bellied Elaenia & $\mathrm{OPCe}$ & ICA & INS/FRU & 8,9 \\
\hline Elaenia Spectabilis & Large Elaenia & $\mathrm{FO}, \mathrm{OP} \mathrm{Ce}$ & AM & INS/FRU & $1,8,9$ \\
\hline Elaenia Chilensis & Chilean Elaenia & OP Ce, OP Ch & ICA & INS & I \\
\hline Elaenia Parvirostris & Small-Billed Elaenia & $\mathrm{OPCe}, \mathrm{OP} \mathrm{Ch}$ & ICA & INS & 5.7 \\
\hline Elaenia Chiriquensis & Lesser Elaenia & $\mathrm{OPCe}$ & ICA & INS & 8.9 \\
\hline Suiriri Suiriri & Suiriri Flycatcher & $\mathrm{OPCe}, \mathrm{OP} \mathrm{Ch}$ & ICA & INS & $4,5,8,9$ \\
\hline Myiopagis Gaimardii & Forest Elaenia & FO & & INS & $1,8,9$ \\
\hline Myiopagis Viridicata & Greenish Elaenia & FO & AM & INS & $\mathrm{I}, 4,5,8,9$ \\
\hline Phaeomyias Murina & Mouse-Colored Tyrannulet & OP Ce, OP Ch & AM & INS & $\mathrm{I}, 4,5,8,9$ \\
\hline Polystictus Pectoralis & Bearded Tachuri & OP Ce, OP Ch & AM & INS & 5 \\
\hline Serpophaga Subcristata & White-Crested Tyrannulet & $\mathrm{OPCe}, \mathrm{OP} \mathrm{Ch}$ & AM & INS & I,4,5,8,9 \\
\hline Legatus Leucophaius & Piratic Flycatcher & $\mathrm{FO}, \mathrm{OP} \mathrm{Ce}, \mathrm{OP} \mathrm{Ch}$ & AM & INS & $1,8,9$ \\
\hline Myiarchus Swainsoni & Swainson's Flycatcher & $\mathrm{FO}, \mathrm{OP} \mathrm{Ce}, \mathrm{OP} \mathrm{Ch}$ & AM & INS & $\mathrm{I}, 4,8,9$ \\
\hline
\end{tabular}


Table Continued...

\begin{tabular}{|c|c|c|c|c|c|}
\hline Taxon & English Name & Habitat & Status & Trophic guild & Surveyed Sites \\
\hline Myiarchus Ferox & Short-Crested Flycatcher & $\mathrm{FO}, \mathrm{OP} \mathrm{Ce}, \mathrm{OP} \mathrm{Ch}$ & & INS & $1,8,9$ \\
\hline Myiarchus Tyrannulus & Brown-Crested Flycatcher & $\mathrm{OP} \mathrm{Ce}, \mathrm{OP} \mathrm{Ch}$ & & INS & $1,2,4,5,6,8,9$ \\
\hline Sirystes Sibilator & Sibilant Sirystes & FO & ICA & INS & 8,9 \\
\hline Casiornis Rufus & Rufous Casiornis & $\mathrm{FO}, \mathrm{OP} \mathrm{Ce}, \mathrm{OP} \mathrm{Ch}$ & AM & INS & $\mathrm{I}, 2,3,4,5,6,7,8,9$ \\
\hline Pitangus Sulphuratus & Great Kiskadee & $\mathrm{AA}, \mathrm{FO}, \mathrm{OP} \mathrm{Ce}, \mathrm{OP} \mathrm{Ch}$ & & ONI & I,2,3,4,5,6,7,8,9 \\
\hline Machetornis Rixosa & Cattle Tyrant & $\mathrm{AA}, \mathrm{OP} \mathrm{Ce}, \mathrm{OP} \mathrm{Ch}$ & ICA & INS & I,2,3,4,5,6,7,8,9 \\
\hline Myiodynastes Maculatus & Streaked Flycatcher & $\mathrm{FO}, \mathrm{OP} \mathrm{Ce}, \mathrm{OP} \mathrm{Ch}$ & AM & INS & $\mathrm{I}, 2,4,5,6,7,8,9$ \\
\hline Megarynchus Pitangua & Boat-Billed Flycatcher & $\mathrm{FO}, \mathrm{OP} \mathrm{Ce}, \mathrm{OP} \mathrm{Ch}$ & ICA & ONI & $1,2,3,4,5,6,7,8,9$ \\
\hline Myiozetetes Cayanensis & Rusty-Margined Flycatcher & $\mathrm{A}, \mathrm{FO}$ & $\mathrm{R}$ & INS & I,4,5,8,9 \\
\hline Myiozetetes Similis & Social Flycatcher & A, FO & $\mathrm{R}$ & INS & 1 \\
\hline Tyrannus Melancholicus & Tropical Kingbird & $\mathrm{AA}, \mathrm{OP} \mathrm{Ce}, \mathrm{OP} \mathrm{Ch}$ & AM & INS & $\mathrm{I}, 2,3,4,5,6,7,8,9$ \\
\hline Tyrannus Savana & Fork-Tailed Flycatcher & $\mathrm{AA}, \mathrm{OP} \mathrm{Ce}, \mathrm{OP} \mathrm{Ch}$ & AM & INS & $\mathrm{I}, 2,4,5,7,8,9$ \\
\hline $\begin{array}{l}\text { Griseotyrannus } \\
\text { Aurantioatrocristatus }\end{array}$ & Crowned Slaty Flycatcher & $\mathrm{AA}, \mathrm{OP} \mathrm{Ce}, \mathrm{OP} \mathrm{Ch}$ & AM & INS & $\mathrm{I}, 2,4,5,6,7,8,9$ \\
\hline Empidonomus Varius & Variegated Flycatcher & OP Ce, OP Ch & AM & INS & $\mathrm{I}, 4,5,6,7,8,9$ \\
\hline Myiophobus Fasciatus & Bran-Colored Flycatcher & $\mathrm{OP} \mathrm{Ce}, \mathrm{OP} \mathrm{Ch}$ & & INS & I,5 \\
\hline Sublegatus Modestus & Southern Scrub-Flycatcher & $\mathrm{OP} \mathrm{Ce}, \mathrm{OP} \mathrm{Ch}$ & AM & INS & I,4,5,8,9 \\
\hline Pyrocephalus Rubinus & Vermilion Flycatcher & $\mathrm{OP} \mathrm{Ce}, \mathrm{OP} \mathrm{Ch}$ & AM & INS & $1,2,4,5,7,8,9$ \\
\hline Fluvicola Albiventer & Black-Backed Water-Tyrant & $A$ & AM & INS & $\mathrm{I}, 2,4,5,6,7$ \\
\hline Arundinicola Leucocephala & White-Headed Marsh Tyrant & $A$ & & INS & $\mathrm{I}, 4,5,6,7,8,9$ \\
\hline Gubernetes Yetapa & Streamer-Tailed Tyrant & $\mathrm{A}, \mathrm{OP} \mathrm{Ce}, \mathrm{OP} \mathrm{Ch}$ & ICA & INS & $1,8,9$ \\
\hline Alectrurus Risora & Strange-Tailed Tyrant & OP Ce, OP Ch & AM & INS & I \\
\hline Cnemotriccus Fuscatus & Fuscous Flycatcher & $\mathrm{FO}, \mathrm{OP} \mathrm{Ce}, \mathrm{OP} \mathrm{Ch}$ & AM & INS & I,4,5,8,9 \\
\hline Hymenops Perspicillatus & Spectacled Tyrant & $\mathrm{OP} \mathrm{Ce}, \mathrm{OP} \mathrm{Ch}$ & AM & INS & 7 \\
\hline Satrapa Icterophrys & Yellow-Browed Tyrant & $\mathrm{OP} \mathrm{Ce}, \mathrm{OP} \mathrm{Ch}$ & AM & INS & $4,5,7$ \\
\hline Xolmis Cinereus & Gray Monjita & $\mathrm{OP} \mathrm{Ce}, \mathrm{OP} \mathrm{Ch}$ & ICA & INS & $\mathrm{I}, 2,4,5,6,7,8,9$ \\
\hline Xolmis Velatus & White-Rumped Monjita & $\mathrm{OP} \mathrm{Ce}, \mathrm{OP} \mathrm{Ch}$ & $\mathrm{R}$ & INS & $1,2,5,6,7,8,9$ \\
\hline Xolmis Irupero & White Monjita & $\mathrm{OP} \mathrm{Ce}, \mathrm{OP} \mathrm{Ch}$ & $\mathrm{R}$ & INS & $\mathrm{I}, 2,4,5,6,7,8,9$ \\
\hline \multicolumn{6}{|l|}{ Vireonidae } \\
\hline Cyclarhis Gujanensis & Rufous-Browed Peppershrike & $\mathrm{FO}, \mathrm{OP} \mathrm{Ce}, \mathrm{OP} \mathrm{Ch}$ & & ONI & $\mathrm{I}, 2,3,4,5,6,7,8,9$ \\
\hline Vireo Chivi & Chivi Vireo & $\mathrm{OP} \mathrm{Ce}, \mathrm{OP} \mathrm{Ch}$ & AM & INS & $\mathrm{I}, 6,7,8$ \\
\hline \multicolumn{6}{|l|}{ Corvidae } \\
\hline Cyanocorax Cyanomelas & Purplish Jay & $\mathrm{FO}, \mathrm{OP} \mathrm{Ce}, \mathrm{OP} \mathrm{Ch}$ & & ONI & $1,2,4,5,7,8,9$ \\
\hline Cyanocorax Cristatellus & Curl-Crested Jay & $\mathrm{OPCe}$ & & ONI & 8,9 \\
\hline Cyanocorax Chrysops & Plush-Crested Jay & $\mathrm{FO}$ & & ONI & $\mathrm{I}, 4,5,7,8,9$ \\
\hline \multicolumn{6}{|l|}{ Hirundinidae } \\
\hline Stelgidopteryx Ruficollis & Southern Rough-Winged Swallow & $\mathrm{A}, \mathrm{FO}$ & ICA & INS & $1,5,8$ \\
\hline Progne Tapera & Brown-Chested Martin & $\mathrm{AA}, \mathrm{OP} \mathrm{Ce}, \mathrm{OP} \mathrm{Ch}$ & ICA & INS & $\mathrm{I}, 2,3,4,5,6,7,8,9$ \\
\hline Progne Chalybea & Gray-Breasted Martin & $\mathrm{AA}, \mathrm{OP} \mathrm{Ce}, \mathrm{OP} \mathrm{Ch}$ & ICA & INS & $1,2,8,9$ \\
\hline Tachycineta Albiventer & White-Winged Swallow & $A$ & & INS & $2,3,4,5$ \\
\hline
\end{tabular}


Table Continued....

\begin{tabular}{|c|c|c|c|c|c|}
\hline Taxon & English Name & Habitat & Status & Trophic guild & Surveyed Sites \\
\hline Tachycineta Leucorrhoa & White-Rumped Swallow & $\mathrm{OP} \mathrm{Ce}, \mathrm{OP} \mathrm{Ch}$ & ICA & INS & $\mathrm{I}, 7$ \\
\hline Riparia Riparia & Bank Swallow & OP Ce, OP Ch & $S$ & & \\
\hline Hirundo Rustica & Barn Swallow & OP Ce, OP Ch & $\mathrm{S}$ & INS & $\mathrm{I}, 7$ \\
\hline \multicolumn{6}{|l|}{ Troglodytidae } \\
\hline Campylorhynchus Turdinus & Thrush-Like Wren & $\mathrm{AA}, \mathrm{FO}, \mathrm{OP} \mathrm{Ce}, \mathrm{OP} \mathrm{Ch}$ & & INS & $\mathrm{I}, 2,3,4,5,6,7,8,9$ \\
\hline Cantorchilus Guarayanus & Fawn-Breasted Wren & $\mathrm{FO}$ & & INS & $\mathrm{I}, 4,5,7,8,9$ \\
\hline \multicolumn{6}{|l|}{ Donacobiidae } \\
\hline Donacobius Atricapilla & Black-Capped Donacobius & $A$ & & INS & $\mathrm{I}, 2,3$ \\
\hline Polioptila Dumicola & Masked Gnatcatcher & OP Ce, OP Ch & & INS & $\mathrm{I}, 2,4,5,6,7,8,9$ \\
\hline \multicolumn{6}{|l|}{ Turdidae } \\
\hline Turdus Leucomelas & Pale-Breasted Thrush & FO & $S$ & ONI & $\mathrm{I}, 4$ \\
\hline Turdus Rufiventris & Rufous-Bellied Thrush & $\mathrm{AA}, \mathrm{FO}, \mathrm{OP} \mathrm{Ce}, \mathrm{OP} \mathrm{Ch}$ & & ONI & $\mathrm{I}, 2,4,5,6,8,9$ \\
\hline Turdus Amaurochalinus & Creamy-Bellied Thrush & $\mathrm{AA}, \mathrm{FO}, \mathrm{OP} \mathrm{Ce}, \mathrm{OP} \mathrm{Ch}$ & $\mathrm{R}$ & ONI & $\mathrm{I}, 2,4,5,6,8,9$ \\
\hline \multicolumn{6}{|l|}{ Mimidae } \\
\hline Mimus Saturninus & Chalk-Browed Mockingbird & $\mathrm{AA}, \mathrm{OP} \mathrm{Ce}, \mathrm{OP} \mathrm{Ch}$ & & ONI & $\mathrm{I}, 2,3,4,5,6,7,8,9$ \\
\hline Mimus Triurus & White-Banded Mockingbird & $\mathrm{AA}, \mathrm{OP} \mathrm{Ce}, \mathrm{OP} \mathrm{Ch}$ & AM & ONI & $\mathrm{I}, 2,7$ \\
\hline \multicolumn{6}{|l|}{ Motacillidae } \\
\hline Anthus Lutescens & Yellowish Pipit & $\mathrm{AA}, \mathrm{OP} \mathrm{Ce}, \mathrm{OP} \mathrm{Ch}$ & $\mathrm{R}$ & INS & $\mathrm{I}, 7$ \\
\hline Zonotrichia Capensis & Rufous-Collared Sparrow & $\mathrm{AA}, \mathrm{OP} \mathrm{Ce}, \mathrm{OP} \mathrm{Ch}$ & & ONI & $\mathrm{I}, 2,8,9$ \\
\hline Ammodramus Humeralis & Grassland Sparrow & $\mathrm{AA}, \mathrm{OP} \mathrm{Ce}, \mathrm{OP} \mathrm{Ch}$ & & GRA & $7,8,9$ \\
\hline Arremon Flavirostris & Saffron-Billed Sparrow & FO & & GRA/INS & 1,5 \\
\hline \multicolumn{6}{|l|}{ Parulidae } \\
\hline Setophaga Pitiayumi & Tropical Parula & $\mathrm{FO}, \mathrm{OP} \mathrm{Ce}, \mathrm{OP} \mathrm{Ch}$ & & INS & $\mathrm{I}, 4,5,8,9$ \\
\hline Geothlypis Aequinoctialis & Masked Yellowthroat & OP Ce, OP Ch & & INS & 7 \\
\hline Basileuterus Culicivorus & Golden-Crowned Warbler & FO & & INS & $\mathrm{I}, 4,5,8,9$ \\
\hline Myiothlypis Flaveola & Flavescent Warbler & FO & & INS & $\mathrm{I}, 4,5,8,9$ \\
\hline \multicolumn{6}{|l|}{ Icteridae } \\
\hline Psarocolius Decumanus & Crested Oropendola & FO & & ONI & $\mathrm{I}, 4,5$ \\
\hline Procacicus Solitarius & Solitary Black Cacique & $\mathrm{FO}, \mathrm{OP} \mathrm{Ce}, \mathrm{OP} \mathrm{Ch}$ & & ONI & $1,2,6,7$ \\
\hline Cacicus Chrysopterus & Golden-Winged Cacique & FO, OP Ce, OP Ch & & ONI & $\mathrm{I}, 2,4,5,6,7,8,9$ \\
\hline Cacicus Haemorrhous & Red-Rumped Cacique & $\mathrm{FO}, \mathrm{OP} \mathrm{Ce}, \mathrm{OP} \mathrm{Ch}$ & & ONI & $1,8,9$ \\
\hline Icterus Pyrrhopterus & Variable Oriole & $\mathrm{FO}, \mathrm{OP} \mathrm{Ce}, \mathrm{OP} \mathrm{Ch}$ & & ONI & $\mathrm{I}, 2,4,5,6,7,8,9$ \\
\hline Icterus Croconotus & Orange-Backed Troupial & $\mathrm{FO}, \mathrm{OP} \mathrm{Ce}, \mathrm{OP} \mathrm{Ch}$ & & ONI & $\mathrm{I}, 2,3,4,5,7$ \\
\hline Gnorimopsar Chopi & Chopi Blackbird & $\mathrm{AA}, \mathrm{OP} \mathrm{Ce}, \mathrm{OP} \mathrm{Ch}$ & & ONI & $\mathrm{I}, 2,3,4,5,6,7,8$ \\
\hline Amblyramphus Holosericeus & Scarlet-Headed Blackbird & $A$ & & ONI & 4,7 \\
\hline Agelasticus Cyanopus & Unicolored Blackbird & A & $\mathrm{R}$ & ONI & $\mathrm{I}, 5,7,8,9$ \\
\hline Chrysomus Ruficapillus & Chestnut-Capped Blackbird & $A$ & $\mathrm{R}$ & ONI & $\mathrm{I}, 2,5,7$ \\
\hline
\end{tabular}


Table Continued....

\begin{tabular}{|c|c|c|c|c|c|}
\hline Taxon & English Name & Habitat & Status & Trophic guild & Surveyed Sites \\
\hline Pseudoleistes Guirahuro & Yellow-Rumped Marshbird & $\mathrm{OP} \mathrm{Ce}$ & & ONI & 8,9 \\
\hline Agelaioides Badius & Grayish Baywing & $\mathrm{OPCe}, \mathrm{OP} \mathrm{Ch}$ & & ONI & $\mathrm{I}, 2,4,5,6,7,8,9$ \\
\hline Molothrus Rufoaxillaris & Screaming Cowbird & $\mathrm{AA}, \mathrm{OP} \mathrm{Ce}, \mathrm{OP} \mathrm{Ch}$ & & ONI & $\mathrm{I}, 2,4,5,7,8,9$ \\
\hline Molothrus Oryzivorus & Giant Cowbird & AA, FO, OP Ce, OP Ch & & ONI & $1,2,7,8$ \\
\hline Sturnella Superciliaris & White-Browed Meadowlark & $\mathrm{AA}, \mathrm{OP} \mathrm{Ce}, \mathrm{OP} \mathrm{Ch}$ & $\mathrm{R}$ & ONI & 7 \\
\hline \multicolumn{6}{|l|}{ Thraupidae } \\
\hline Paroaria Coronata & Red-Crested Cardinal & $\mathrm{AA}, \mathrm{FO}, \mathrm{OP} \mathrm{Ce}, \mathrm{OP} \mathrm{Ch}$ & & GRA & $\mathrm{I}, 2,3,4,5,6,8,9$ \\
\hline Paroaria Capitata & Yellow-Billed Cardinal & AA, OP Ce, OP Ch & & GRA & $\mathrm{I}, 2,3,4,5,6,7,8,9$ \\
\hline Tangara Palmarum & Palm Tanager & AA, FO, OP Ce, OP Ch & & FRU & $1,2,4,5$ \\
\hline Tangara Cayana & Burnished-Buff Tanager & $\mathrm{FO}, \mathrm{OP} \mathrm{Ce}, \mathrm{OP} \mathrm{Ch}$ & & FRU & $\mathrm{I}, 2$ \\
\hline Nemosia Pileata & Hooded Tanager & $\mathrm{FO}, \mathrm{OP} \mathrm{Ce}, \mathrm{OP} \mathrm{Ch}$ & & INS & $\mathrm{I}, 4,5$ \\
\hline Conirostrum Speciosum & Chestnut-Vented Conebill & $\mathrm{FO}, \mathrm{OP} \mathrm{Ce}, \mathrm{OP} \mathrm{Ch}$ & ICA & INS & $\mathrm{I}, 4,5,6,8,9$ \\
\hline Sicalis Flaveola & Saffron Finch & OP Ce, OP Ch & & GRA & $\mathrm{I}, 2,3,4,5,6,7,8,9$ \\
\hline Hemithraupis Guira & Guira Tanager & $\mathrm{FO}, \mathrm{OP} \mathrm{Ce}, \mathrm{OP} \mathrm{Ch}$ & & INS & 8,9 \\
\hline Volatinia Jacarina & Blue-Black Grassquit & $\mathrm{AA}, \mathrm{OP} \mathrm{Ce}, \mathrm{OP} \mathrm{Ch}$ & $\mathrm{R}$ & GRA & $\mathrm{I}, 4,5,6,7,8,9$ \\
\hline Eucometis Penicillata & Gray-Headed Tanager & $\mathrm{FO}$ & & INS/FRU & $\mathrm{I}, 4,5,8,9$ \\
\hline Coryphospingus Cucullatus & Red-Crested Finch & $\mathrm{AA}, \mathrm{FO}, \mathrm{OP} \mathrm{Ce}, \mathrm{OP} \mathrm{Ch}$ & & GRA & $\mathrm{I}, 2,4,5,7,8,9$ \\
\hline Tachyphonus Rufus & White-Lined Tanager & $\mathrm{FO}, \mathrm{OP} \mathrm{Ce}, \mathrm{OP} \mathrm{Ch}$ & & FRU & $\mathrm{I}, 2,3,5,6,8,9$ \\
\hline Tersina Viridis & Swallow-Tanager & FO & ICA & INS/FRU & 8 \\
\hline Coereba Flaveola & Bananaquit & $\mathrm{AA}, \mathrm{FO}, \mathrm{OP} \mathrm{Ce}, \mathrm{OP} \mathrm{Ch}$ & & NEC/INS & $1,2,6$ \\
\hline Sporophila Lineola & Lined Seedeater & OP Ce, OP Ch & ICA & GRA & $\mathrm{I}, 2,6,7$ \\
\hline Sporophila Collaris & Rusty-Collared Seedeater & $\mathrm{OPCe}, \mathrm{OP} \mathrm{Ch}$ & ICA & GRA & $\mathrm{I}, 2,4,5,6,7,8$ \\
\hline Sporophila Caerulescens & Double-Collared Seedeater & OP $\mathrm{Ce}, \mathrm{OP} \mathrm{Ch}$ & $\mathrm{R}$ & GRA & $1,2,6,7$ \\
\hline Sporophila Leucoptera & White-Bellied Seedeater & OP Ce, OP Ch & & GRA & $\mathrm{I}, 2,4,5,6,7$ \\
\hline Sporophila Hypoxantha & Tawny-Bellied Seedeater & OP $\mathrm{Ce}, \mathrm{OP} \mathrm{Ch}$ & $\mathrm{R}$ & GRA & 7 \\
\hline Sporophila Ruficollis & Dark-Throated Seedeater & OP Ce, OP Ch & AM & GRA & 7 \\
\hline Sporophila Palustris & Marsh Seedeater & OP Ce, OP Ch & AM & GRA & 7 \\
\hline Sporophila Angolensis & Chestnut-Bellied Seed-Finch & $\mathrm{FO}, \mathrm{OP} \mathrm{Ce}, \mathrm{OP} \mathrm{Ch}$ & & GRA & $\mathrm{I}, 4,5,8,9$ \\
\hline Emberizoides Herbicola & Wedge-Tailed Grass-Finch & $\mathrm{OPCe}, \mathrm{OP} \mathrm{Ch}$ & & INS & 8,9 \\
\hline Saltatricula Atricollis & Black-Throated Saltator & $\mathrm{OP} \mathrm{Ce}$ & & INS & 8,9 \\
\hline Saltatricula Multicolor & Many-Colored Chaco Finch & $\mathrm{OP} \mathrm{Ch}$ & & INS & 2 \\
\hline Saltator Coerulescens & Grayish Saltator & $\mathrm{OP} \mathrm{Ce}, \mathrm{OP} \mathrm{Ch}$ & & ONI & $\mathrm{I}, 2,3,4,5,6,7,8,9$ \\
\hline Saltator Similis & Green-Winged Saltator & $\mathrm{FO}$ & $\mathrm{R}$ & ONI & I \\
\hline Microspingus Melanoleucus & Black-Capped Warbling-Finch & $\mathrm{OP} \mathrm{Ce}, \mathrm{OP} \mathrm{Ch}$ & & INS & $\mathrm{I}, 2,4,5,6,7,8,9$ \\
\hline \multicolumn{6}{|l|}{ Cardinalidae } \\
\hline Piranga Flava & Hepatic Tanager & OP Ce, OP Ch & AM & INS/FRU & 4 \\
\hline Cyanoloxia Brissonii & Ultramarine Grosbeak & OP Ce, OP Ch & & ONI & 5 \\
\hline
\end{tabular}


Table Continued....

\begin{tabular}{|c|c|c|c|c|c|}
\hline Taxon & English Name & Habitat & Status & Trophic guild & Surveyed Sites \\
\hline \multicolumn{6}{|l|}{ Fringillidae } \\
\hline Spinus Magellanicus & Hooded Siskin & $\mathrm{FO}, \mathrm{OP} \mathrm{Ce}, \mathrm{OP} \mathrm{Ch}$ & & GRA & 4,8 \\
\hline Euphonia Chlorotica & Purple-Throated Euphonia & $\mathrm{FO}, \mathrm{OP} \mathrm{Ce}, \mathrm{OP} \mathrm{Ch}$ & & FRU & $\mathrm{I}, 2,4,5,7,8,9$ \\
\hline \multicolumn{6}{|l|}{ Passeridae } \\
\hline
\end{tabular}

Some of our recorded species were only recently recorded for the Pantanal wetland, including: Nothura boraquira, Aramides ypecaha, Pardirallus maculatus, Calidris himantopus, Dendrocolaptes platyrostris, Elaenia chilensis, E. parvirostris, Platyrinchus mystaceus, Hymenops perspicillatus, Pachyramphus validus, Xenopsaris albinucha, Pseudoleites guirahuro, Sporophila ruficollis, $S$. palustris. ${ }^{10}$ A considerable number of species migrate to the Pantanal wetland, ${ }^{32}$ and others are present in the Pantanal in Cerrado vegetation. ${ }^{26}$ Species such as Cyanocorax cristatellus and Saltatricula atricollis, common in open areas associated with Cerrado vegetation, were found in this study in similar habitats, showing the transitional feature of the Porto Murtinho region. There are migrant species that occur in the region. The Tyrannidae Austral migrants, for example, arrive in general by the end of the dry season, fixing home ranges in the region throughout the wet season, coinciding with greater abundance of food. ${ }^{33,34}$ Some of the species registered deserve special attention since they are officially listed as threatened under national and international guidelines, ${ }^{35,36}$ considering the alteration and loss of their natural habitats. When dealing with threatened species, the value of rarity is one trophy for the birders, which requires a fair conservation strategy in order to balance protection and visiting strategies. ${ }^{37}$ On the other hand, the number of threatened species found in this study reinforces the need for a reliable conservation strategy. Natural habitats have been modified and the increasing deforestation has reached the study area. ${ }^{5}$ There are no protected areas designated for the Porto Murtinho region to preserve the native Chaco natural habitats and their associated biodiversity. All activities in the region related to the exploitation of biodiversity resources have been unsustainable in recent years, for example the use of the red quebracho (S. balansae) to extract tannin, the leaves of erva mate (Ilex paraguariensis) for tea, as well as predatory fisheries. In addition to the intrinsic value of biodiversity, its conservation needs to consider a combination of multiple strategies. For example, biodiversity plays an important role to human well-being and health, providing basic products and ecosystem services. Thus, conservation is also related to the social and economic dimensions of development. But although this relationship, human land occupation has become the dominant force that negatively impacts the biological system. The avifauna of the Chaco expresses the fascination of the local people, who have generated stories and folklore associated with mystic birds, aggregating cultural heritage. The knowledge on birds can improve not only scientific support but ways to develop sustainable tourism in the region. The benefits of nature-based tourism to biodiversity conservation are often rarely to profitability of local environmentallybased rational use to achieve sustainability. The protection of biodiversity is essential in the fight to reduce local human poverty and to achieve sustainable development. The challenge is to pursue responsible travel to relatively undisturbed natural area, such as the Brazilian portion of Chaco, with the specific objective of studying, admiring, and enjoying the scenery and its wildlife, as well as any existing cultural manifestations found in the region. However, in its broadest sense, nature-based tourism has to be sustainable in terms of its potential negative effects on the environment, for its successful conduct. ${ }^{38}$

\section{Conclusion}

The present results contribute to knowledge of the great diversity of bird species in the region, which may be an attraction for sustainable tourism through birdwatching activities. This should be an incentive to protect the avifauna and their habitats, achieving conservation for the biodiversity of the region.

\section{Acknowledgements}

We are thankful to the Prefeitura Municipal de Porto Murtinho, to the Brazilian Army- $2^{\text {a }}$ Cia Fronteira, and to Fabio Santos for all the support received during the field work, especially during the activities 2008-2015. Flavia Batista helped to elaborate the map.

\section{Conflict of interest}

No conflict of interest was detected during the work carried out in the field or for its publication.

\section{References}

1. Constanza R, Groot R, Sutton P, et al. Changes in the global value of ecosystem services. Global environmental change. 2014;26:152-158.

2. Steven R, Morrison C, Castley JG. Birdwatching and avitourism: a global review of research into its participant markets, distribution and impacts, highlighting future research priorities to inform sustainable avitourism management. Journal of Sustainable Tourism. 2014;23(8-9):1257-1276.

3. Prado DE. What is the Gran Chaco vegetation in South America? I. A review. Contribution to the study of flora and vegetation of the Chaco V. Candollea. 1993;48(1):145-172.

4. Sarmiento G. Ecological and floristic convergences between seasonal plant formations of Tropical and Subtropical South America. Journal of Ecology. 1972;60(2):367-410.

5. Silva MP, Mauro RA, Abdon M, et al. State of Conservation of the Chaco (Brazilian savannah). In: Faleiro FG, Farias-Neto AL, editors. Proceedings of the IX National Symposium Cerrado. Brazil: Embrapa Cerrados; 2008. p. 1-6.

6. Pott A, Oliveira AKM, Damasceno Junior GA, et al. Plant diversity of the Pantanal wetland. Braz J Biol. 2011;71(1):65-273.

7. Short LL. A zoogeographic analysis of the South American Chaco avifauna. Bulletin of the AMNH. 1975;154(3):163-352.

8. Prado DE, Gibbs PE, Pott A, et al. The Chaco-Pantanal transition in southern Mato Grosso, Brazil. In: Furley PA, Proctor J Ratter JA, editors. Natureand dynamics of forest-savanna boundaries. England: Chapman \& Hall; 1992. p. 451-470.

9. Tubelis DP, Tomas WM. Birds species of the Pantanal wetland, Brazil. Ararajuba. 2003;11(1):5-37. 
10. Nunes AP. Quantas especies de aves ocorrem no Pantanal brasileiro Atualidades Ornitologicas. 2011;1-10.

11. Straube FC, Urben Filho A, Pivatto MAC, et al. Nova contribuicao a ornitologia do Chaco brasileiro (Mato Grosso do Sul, Brasil). Atualidades ornitologicas. 2006:1-27.

12. Pacheco JF, Bauer C. A colecao de aves preparadas por Adolf Schneider em Porto Quebracho, Mato Grosso do Sul, Brasil, em 1941. Notulas Faunisticas. 1994;64:1-7.

13. Carvalho FS, Sartori ALB. Reproductive phenology and seed dispersal syndromes of woody species in the Brazilian Chaco. Journal of Vegetation Science. 2014;26(2):302-311.

14. Piacentini VQ, Aleixo A, Agnes CE, et al. Annotated checklist of the birds of Brazil by the Brazilian Ornithological Records Committee. Brazilian Journal of Ornithology. 2015;23(2):90-298.

15. Sick H. Introducao a ornitologia brasileira. Brazil: Nova Fronteira; 1997. 912 p.

16. Codesido M, Bilenca D. Variacion estacional de un ensamble de aves enun Bosque Subtropical Semiarido del Chaco Argentino. Biotropica. 2004;36(4):544-554

17. Kratter AW, Sillett TS, Chesser RT, et al. Avifauna of a Chaco locality in Bolivia. Wilson Bull. 1993;105(1):114-141.

18. Zyskowski K, Robbins MB, Peterson AT, et al. Avifauna of the northern Paraguayan Chaco. Ornitologia neotropical. 2003;14:247-262.

19. Silotti RY. The Encyclopedia of Brazilian Birds. Wiki Aves. 2015

20. Cracraft J. Historical biogeography and patterns of differentiation within the South American avifauna:areas of endemism. Ornithological Monographs. 1985;36:49-84.

21. Delhey KV, Scorolli AL. Abundancia relativa y estatus de residenciadelpepiterochico (Saltatricula multicolor) enel sudoeste de laProvincia de Buenos Aires, Argentina. El Hornero. 2002;17(1):41-44.

22. Herzog SK, Kessler M. Biogeography and composition of dry forest bird communities In Bolivia. Journal fur Ornithologie. 2002;143(2):171-204.

23. Straube FC, Di Giacomo A. A avifauna das regioes subtropical e temperada do Neotropico:desafios biogeograficos. Cieencia\& Ambiente. 2007;35:137-166

24. Silva JMC. Biogeographic analysis of South American Cerrado avifauna Steenstrupia. 1995;21:49-67.
25. Pivatto MAC, Manco DG, Straube FC, et al. Aves do Planalto da Bodoquena, Estado do Mato Grosso do Sul (Brasil). Atualidades Ornitologicas. 2006;129:1-26.

26. Pivatto MAC, Donatelli RJ, Manco DG. Aves da Fazenda Santa Emília, Aquidauana, Mato Grosso do Sul. Atualidades Ornitologicas. 2008;143:33-37.

27. Godoi MN, Souza EO. The effects of forest-savanna-grassland gradients on bird communities of Chiquitano Dry Forests domain, in western Brazil. An Acad Bras Cienc. 2016;88(3):1755-1767.

28. Herzog SK, Auza RWS, Hennessey AB. Patronesecorregionales de riqueza, endemismo y amenaza de laavifauna boliviana:prioridades para laplanificacionecorregional. Ecologia en Bolivia. 2005;40(2):27-40.

29. Werneck FP. The diversification of eastern South American open vegetation biomes:historical biogeography and perspectives. Quaternary Science Reviews. 2011;30(13):1630-1648.

30. The Nature Conservancy. Evaluacion Ecorregional del Gran Chaco Americano / Gran Chaco Americano Ecoregional Assessment. 1st ed. Argentina: Fundacion Vida Silvestre; 2005. 24 p.

31. Alho CJR, Sabino J. Seasonal Pantanal flood pulse: implications for biodiversity conservation - a review. Oecologia Australis. 2012;16(4):958-978.

32. Nunes AP, Tomas WM. Aves migratórias ocorrentes no Pantanal: caracterização e conservação. Brazil: Embrapa Pantanal; 2004. 27 p.

33. Chesser RT. Migration in South America: an overview of the austral system. Bird Conservation International. 1994;4(2-3):91-107.

34. Hayes FE, Scharf PA, Ridgely RS. Austral bird migrants in Paraguay. The Condor. 1994;96(1):83-97.

35. Ministry of the Environment. Official national list of endangered species of fauna. Brazil: Official Gazette of the Union; 2014.

36. The IUCN Red List of Threatened Species. 2017.

37. Booth JE, Gaston KJ, Evans KL, et al. The value of species rarity in biodiversity recreation: a birdwatching example. Biological Conservation. 2011;144:2728-2732.

38. Silva JSV, Caputo ACB. Localizacao e distribuicao da vegetacao savana estepica (Chaco) no Pantanal brasileiro. Brazil: Anais $3^{\circ}$ Simposio de Geotecnologias no Pantanal, Caceres, MT, Embrapa Informática Agropecuaria/INPE; 2010. p. 314-323. 